Rolf Bergmann

\title{
KULTURGESCHICHTLICHE ASPEKTE DES ALTHOCHDEUTSCHEN GLOSSENWORTSCHATZES
}

\section{Einl eitung}

Die denkbaren theoretischen Problematisierungen der Begriffe Kulturgeschichte, althochdeutsch, Glosse und Wortschatz unterbleiben hier aus Platzgründen; für Kulturgeschichte und Wortschatz darf insbesondere auf den Kontext des ganzen Tagungsbandes verwiesen werden. In der Tradition der Glossenforschung (vgl. H. Thoma 1958; R. Schützeichel 1982, 1985, 1991; N. Henkel 1997) wird unter dem Glossenwortschatz einfach die Gesamtheit der in den Glossen und Glossaren bezeugten Lexeme verstanden, unter althochdeutschem Glossenwortschatz die Ü berlieferung des 8. bis 11. J ahrhunderts und die in ihrer Tradition stehende jüngere Glossarüberlieferung.

Die neuesten, auch methodisch reflektierten Zahlenangaben zum althochdeutschen Wortschatz bietet der Artikel ,Lexikologie und Lexikographie des Althochdeutschen' von J. Spl ett (2000). Demnach umfasst der bezeugte althochdeutsche Wortschatz etwa 28.500 Lexeme, von denen 4.400 nur in der Textüberlieferung vorkommen, 6.500 in der Textüberlieferung und der Glossenüberlieferung gemeinsam und 17.600 nur in der Glossenüberlieferung. Das bedeutet, dass knapp $62 \%$ oder fast zwei Drittel aller althochdeutschen Lexeme nur durch die Glossenüberlieferung bezeugt sind.

\begin{tabular}{|l|l|}
\hline nur in Texten: 4.400 & \multirow{2}{*}{ in Texten: 10.900} \\
\hline in Texten und Glossen: 6.500 & \\
\hline nur in Glossen: 17.600 & in Glossen: 24.100 \\
\hline Gesamtüberlieferung: 28.500 & \\
\hline
\end{tabular}

Die quantitative B edeutung der Glossen wird noch dadurch unterstrichen, dass mehr als die Hälfte der 10.000 in Texten überlieferten Wörter auch in Glossen überliefert ist, so dass schließlich für 24.100 von 28.500 Lexemen (=ca. $85 \%$ ) die Glossenüberlieferung relevant ist. 
Zur besonderen Relevanz des Glossenwortschatzes im Allgemeinen gehört auch die Zahl der darin enthaltenen Hapaxlegomena. Sie kann zwar beim derzeitigen lexikographischen Arbeitsstand noch nicht insgesamt beziffert werden, doch vermittelt die Zahl von knapp $700 \mathrm{H}$ apaxlegomena unter den 3.682 Lexemen des Abrogans-Glossars eine gewisse Vorstellung, auch wenn man diesen prozentualen Anteil von $19 \%$ sicher nicht auf den ganzen Glossenwortschatz wird hochrechnen dürfen.

Die F rage nach kulturgeschichtlichen Aspekten des althochdeutschen Wortschatzes, speziell des in den Glossen überlieferten, gehört in den Forschungsbereich der historischen Lexikologie als Teil der Sprachgeschichtsforschung und der Traditionsforschung insgesamt, wie 0 . R eichmann in dem HSK -Band ,Sprachgeschichte' feststellt (R eichmann/W ol f 1998). Gegenstand der historischen Lexikologie ist nach O. Reichmann „all dasjenige, was Menschen im Laufe der Kulturgeschichte an Sachgütern, an gesellschaftlichen Einrichtungen, an Erkenntnissen, Ideologien und $\mathrm{H}$ andlungen für so wichtig erachtet haben, daß es im Wortschatz der Sprache einen Niederschlag gefunden hat. Noch schärfer formuliert: Es gibt in der Kulturgeschichte nichts Relevantes, das (in realistischer Ausdrucksweise) nicht mit lexikalischen Mitteln bezeichnet worden wäre." (S. 611)

$\mathrm{H}$ istorische Lexikologie ist am althochdeutschen Wortschatz zumeist als onomasiologische Forschung in der Tradition der Forschungsrichtung ,Wörter und Sachen' betrieben worden (R. Schmidt-W iegand 1975, 1981; R. Gr osse/l. K öppe 2000). Im Folgenden soll zunächst ein Überblick über den Forschungsstand gegeben werden, was wegen des Fehlens einer entsprechenden Bibliographie dringend nötig ist. Anschließend soll exemplarisch veranschaulicht werden, welche große Rolle der Glossenwortschatz bei dieser Forschung spielt. Schließlich ist wenigstens kurz auf ganz bestimmte charakteristische methodische Probleme dieser Forschungsrichtung einzugehen.

\section{Onomasiol ogische For schung zum Al thochdeutschen im Ü ber bl ick}

Es erscheint nicht ganz einfach, onomasiologische Forschung zu einer sprachhistorischen E poche überblicksartig zu ordnen. Das Begriffssystem von R. Hallig und W. von W artburg (1963) und die Gliederung des Dornseiff sind schon an sich problematisch genug und würden in der Anwendung auf das deutsche Frühmittelalter noch problematischer. Hinzu kommt, dass die Forschungslage selbst sehr zufällig wirkt; neben 
zusammenfassenden Darstellungen ganzer Sachbereiche größeren oder kleineren U mfangs wie etwa M. H eynes ,F ünf Bücher[n] deutscher Hausaltertümer' stehen ausgesprochen spezielle Arbeiten zu Einzelphänomenen. Trotzdem sei der Versuch unternommen, etwas Ordnung herzustelIen und einen Überblick zu geben, der aber sicher noch ergänzungsfähig ist.

Im Bereich Tiere und Pflanzen sind zu nennen die Arbeiten zu den Säugetiernamen von H. Pal ander (1899) sowie zu den Tierbezeichnungen in bestimmten Handschriften die freilich nicht onomasiologische Arbeit von E. N euss (1973). Von H. Pal ander stammt auch die unter dem Namen Suol ahti (1909) erschienene umfangreiche Arbeit zu den Vogelnamen. Für die Pflanzennamen gibt es die Studien von E. B j ör kman (1902, 1904[05), zu den Heilpflanzennamen die von H.-R. F ehI mann (1982). Im Bereich der Naturphänomene sind noch E. R ooth s Spezialuntersuchung der Bezeichnungen für den Eiszapfen (1961), E. M einekes Bernstein-Arbeit (1984) sowie A. Löt scher s Schallwörter-Studie (1973) anzuführen.

Angeschlossen sei der Bereich der Landwirtschaft mit der älteren, übergreifenden Arbeit von G. B aur ( 1960), spezielleren Arbeiten von R. B er g mann (1975) und J. B r andsch (1987) zu den Bezeichnungen für Bauer und Hofgesinde, von $\mathrm{H}$. Tief enbach zu den Bezeichnungen für Fluren (1980b) sowie für Mist und Dünger (1980a), von R. Schmidt -W iegand zu den Bezeichnungen des Pfluges (1981) und von E. A l anne zur W einbauterminologie (1950).

Den W ortschatz des Bereichs Nahrung hat T. Zanier (1989/90) untersucht, den des Bereichs Nahrungszubereitung jüngst $A$. M ikel eit is-W inter (2001).

Zum Medizin-Wortschatz sind Arbeiten von A. Nieder hel I mann $(1981,1983)$ sowie von J. R iecke $(2000,2003)$ anzuführen, insbesondere seine Gießener Habilitationsschrift (2004).

Zum Hausbau ist eine Untersuchung von M. Hil ker-Suckrau (1981) zu nennen, zu Werkzeugbezeichnungen im Bauhandwerk wieder ein Beitrag von H. Tief enbach (1983), zu Gefäßbezeichnungen die Dissertation von J. Rohr (1909). Zum Gerät im weiteren Sinne seien hier auch die W affen gezählt, für die die ältere Arbeit von E. M aschke (1926) anzuführen ist sowie mehrere Arbeiten von D. H üpper [-D r öge] $(1981,1983,1986)$ zu den Bezeichnungen für Schutz- und Angriffswaffen. $F u ̈ r$ die Bezeichnungen von Münzen liegen die Studien von E. Schr öder (1918a,b) vor, für Musikinstrumente die Arbeit von W. Relleke (1980). Das Sachgebiet Verkehr wird in Arbeiten von L. V oet z (1989) zu Wegebezeichnungen und von H. Tief enbach (1989) zu Furten berührt, das Sachgebiet Verwaltung 
in M. Gebhardt s Darstellung der Verwaltung des königlichen Hofes im F rühmittelalter und ihrer Widerspiegelung in den althochdeutschen Glossen (1985). Mit D. H üpper -D röges Arbeit zu Textsortenbezeichnungen (1986) ist das Gebiet der Schriftlichkeit vertreten, mit ihrer Untersuchung der Bezeichnungen im Umfeld des gerichtlichen Zweikampfes (1984) das Rechtswesen; dazu gehört auch H.H. M unsk es Untersuchung des Rechtswortschatzes im Bereich der Missetaten (1973).

Der Mensch ist Thema des Buches ,Frau' und ,Mann' von B. Kochskämper (1999) sowie der ins Mittelhochdeutsche ausgreifenden Darstellung der ,German Kinship Terms' (1990) von W. J . J ones. Die Bezeichnungen für soziale Stände, Schichten und Gruppen sowie für ,F reie, Nachbarn und Gefolgsleute' hat G. von Ol ber g untersucht $(1983,1991)$, die Wörter für ,Herr' G. Ehr ismann (1905/06) und D. H. Gr een (1965), die Bezeichnungen für ,Herr sein' und ,Knecht sein' F. Mezger (1930) und Handwerkerbezeichnungen L. V oet z (1983).

Mit A. W aags ,Bezeichnungen des Geistlichen' (1931[32) ist der Bereich der Religion angesprochen. Zu den heidnischen Bezeichnungen der Kultstätte und zu Zauber und W eissagung und zum Heidentum insgesamt gibt es die Arbeiten von H. W esche (1932, 1937, 1940), zu den Bezeichnungen des christlichen Gotteshauses die von A. M asser (1966). Den althochdeutschen Wortschatz auf dem Gebiet der Weissagung hat $\mathrm{H}$. de B oor (1944) behandelt, die Wörter für Himmel und Hölle R. H och hol zer (1996), die Bezeichnungen der sieben Todsünden W. van A cker en (1904), das Wortfeld der Superbia Ch. St ait i (2000), den kirchensprachlichen Bereich in der Wiedergabe von gloria, temptatio, conversio K. F. Fr eudenthal (1959) und in der Wiedergabe von tristitia D. Rupr echt (1959). Von $G$. de Smet sind zahlreiche Studien zu den Bezeichnungen für, leiden' und das Leiden Christi $(1953,1962)$, für Auferstehen und Auferstehung (1961), für remissio peccatorum (1973) usw. anzuführen. Ein Artikel von M. R ein ,Zur Bedeutungsbreite des Wortes bekehren und zur Bezeichnungskonkurrenz im Rahmen des Konzeptes >bekehren< im früheren Althochdeutschen' (2001) gibt bereits im Titel ein moderneres methodisches Verständnis zu erkennen.

Zum Intellektualwortschatz ist an die berühmte Arbeit von J. Trier (1931) zu erinnern, zum Begriffsfeld des Zweifels eine Dissertation von L. Paul y (1944) zu nennen, zu Notkers Wortschatz auf dem Gebiet des Fühlens eine Dissertation von P. K I opsch (1955) und zu ,Geist' und ,Seele' die Untersuchung von G. B ecker (1964).

In all diesen Arbeiten werden onomasiologische und semasiologische Vorgehensweise kombiniert, insofern bei einem onomasiologischen Aus- 
gangspunkt die Einzellexeme notwendigerweise auch semasiologisch behandelt werden. Deshalb ist es zweckmäßig, in eine onomasiologische Bibliographie und Übersicht auch semasiologische Untersuchungen aufzunehmen, sofern sie auch unter onomasiologischem Aspekt zu benutzen sind wie etwa $B$. Meinekes Untersuchung der Wörter chind und barn (1987) oder C. L e cout eux s Arbeiten zu Schrat (1985) und H exe (1983).

\section{Zur Relevanz des Glossenwortschatzes in der onomasiol ogischen For schung}

Nicht wenige Untersuchungen nennen im Titel explizit die Glossen als Quellen, womit in der Regel keine Einschränkung gegenüber der Textüberlieferung vorgenommen, sondern die Wichtigkeit der Glossen unterstrichen werden soll, so zum Beispiel bei G. Baur, Der Wortschatz der Landwirtschaft im Bereich der ahd. Glossen; E. Björ kman, Die Pflanzennamen der althochdeutschen Glossen; E. M aschke, Studien zu Waffennamen der althochdeutschen Glossen; J . R ohr, Die Gefäße in den althochdeutschen Glossen usw.

In einer Reihe übergreifender, die gesamte althochdeutsche Überlieferung auswertender Arbeiten finden sich detaillierte Übersichten über die herangezogenen Belegstellen, die eine genaue $V$ orstellung ihrer jeweiligen Relevanz für die Onomasiologie des betreffenden Sachgebiets vermitteln. So bietet R. Hochhol zer in seiner Arbeit ,Himmel und Hölle' ein Belegverzeichnis (1996, S. 195[209), B. K ochskämper (1999) unterscheidet in jedem ihrer Wortartikel zu ,Frau' und ,Mann' Textbelege und Glossenbelege und A. M ik el eit is-W int er gibt zu den beiden Großkapiteln ,Bezeichnungen für Tätigkeiten' und ,Bezeichnungen für Personen' eine tabellarische Übersicht mit Kommentar zur Verteilung der Bezeichnungen in den Quellen (2001, S. 232f. und S. 311). In diesen Tabellen ist unmittelbar erkennbar, dass Bezeichungen für Personen im Bereich Nahrungszubereitung überhaupt nur in Glossen überliefert sind, Bezeichnungen für Tätigkeiten überwiegend dort.

Die Quellenlage ist in den Einzeluntersuchungen natürlich am Belegmaterial unmittelbar erkennbar; sie wird aber auch reflektiert und explizit angesprochen. Ein sehr anschauliches und hier weiterführendes Beispiel gibt H. Su ol aht is Einleitung zu seinen, Vogelnamen' (1909, S. V):

Für eine geschichtliche Untersuchung der deutschen Vogelnamen bieten die zusammenhängenden, fast ausschließlich religiös gefärbten Texte der ersten literarischen Sprachperiode 
nur äußerst dürftiges Belegmaterial. Um so wichtiger ist der in den Glossen aufgespeicherte W ortschatz, welcher in dem großen Sammelwerke von Steinmeyer und Sievers dem Forscher bequem zugänglich ist. Freilich hat die kirchliche Richtung jener Zeit auch auf die lexikalische Arbeit inren Stempel aufgedrückt, so daß von den gesamten Glossen die zu der Bibel und anderen religiösen Schriften gehörigen einen besonders breiten Raum einnehmen. Aber unter innen befinden sich doch auch die vielen Vogelnamen, welche im Leviticus $(11,17)$ und Deuteronomium $(14,17)$ aufgezählt werden und die uns also in zahlreichen Übersetzungen überliefert sind. Auch von den Schriften der profanen Schriftsteller sind einige gut glossiert worden und aus dem Charakter in den Werken des Vergilius, Prudentius u.a. folgt, daß manche Vogelnamen unter den Glossen vorkommen. Das reichhaltigste Material enthalten jedoch die alphabetisch und sachlich geordneten Glossare, von denen das unter dem Namen Summarium Heinrici bekannte spätalthochdeutsche Gruppenglossar und die etwas früher entstandenen Glossen zu einem hexametrischen Verzeichnis der Tiere und Pflanzen besonders ausgiebig sind.

In dieselbe Richtung weist $\mathrm{H}$. Tief enbachs lakonische Feststellung am Anfang seiner entsprechenden Untersuchung: „Sprachliche Belege für den Mist sind im Althochdeutschen in einer angesichts der Thematik der Texte [zu ergänzen: und Glossen] erstaunlichen Vielzahl vorhanden." (1980a, S. 45).

Damit kommen die Quellen selbst differenzierter in den Blick.

\section{Quel I enkundl iche Aspekte}

Der Quellenbereich der althochdeutschen Glossen wird zunächst eingeteilt in Textglossierungen und Glossare (vgl. R. B er g mann 1997, 2000a, 2000b, 2003; E. Glaser 2003; R. Bergmann/E. Glaser/C. Moul in-F ankhänel [H rsg.] 2001 jeweils mit weiterer Literatur). Glossare sind entweder als Sachglossare auf Spezialgebiete bzw. auf das gesamte Wissen bezogen oder als Textglossare auf einen einzelnen Text. Textglossare sind meist nach der Reihenfolge des Auftretens der Wörter im Text angeordnet, manchmal auch alphabetisch; in beiden Anordnungsformen gibt es zum Beispiel Bibelglossare. Sachglossare begegnen in sachlicher Anordnung wie das Summarium Heinrici, in alphabetischer Anordnung wie die Glossae Salomonis und viele Spezialglossare etwa von Pflanzenbezeichnungen und schließlich in der mnemotechnisch begründeten Organisationsform der Hexameter wie in den Versus de volucribus, besti is, arboribus, piscibus, vermibus, canibus, herbis, membris humanis, die dem Erlernen lateinischer Vokabeln dienten. Auf einen einleitenden Vers, der das betreffende Sachgebiet nennt, folgen ohne syntaktischen Zusammenhang in lediglich metrischer Anordnung die betreffenden Einzelwörter, gelegentlich durch et 
oder cum verbunden (E. St einmeyer /E. Siever S III, 1895, S. 49, Anm. 3; S. 47, Anm. 9). So beginnen im clm 614 beispielsweise die F ischverse so:

Hic etiam disces sapidos discernere pisces.

Hier sollst du nun lernen die essbaren Fische zu unterscheiden.

Lucius et mullus lupus allec truta timallus

Hainio cum squilla. scorpio cum scelpia.

Für die Vogelverse lautet der Eingangsvers, hier zitiert nach clm 23496, f.10ra:

Hic uolucres çeli referam sermone fideli

Hier will ich die Vögel des Himmels in zuverlässiger F ormulierung aufzählen.

Sehr oft sind die Verse in abgesetzten Zeilen mit großem Zeilenabstand geschrieben und die deutschen Äquivalente interlinear über den lateinischen Lemmata eingetragen, so dass das zu erlernende Vokabelwissen in zweisprachiger doppelzeiliger Anordnung funktional organisiert ist, ganz entsprechend zweispaltiger Anordnung in zweisprachigen Wörterbüchern.

Die wortgeschichtliche Bedeutung dieser Versus-Glossen lässt sich an einigen von St. St ricker (2001, S. 550) zusammengestellten Zahlen leicht erkennen: Die Versus de volucribus beispielsweise umfassen $22 \mathrm{H}$ exameter mit 72 Vogelbezeichnungen; die Gesamtzahl der deutschen Glossen zu den Versus erreicht in manchen Handschriften 300, die Gesamtzahl volkssprachiger Belege in allen Handschriften beträgt annähernd 10.000. Von den über 70 bei $\mathrm{H}$. Suol ahti behandelten bereits althochdeutsch bezeugten Vogelbezeichnungen sind hingegen nur knapp 20 auch in literarischen Texten bezeugt.

Unter den allgemeinen Sachglossaren sei wegen des besonders hohen Alters der in die zweite Hälfte des 8. J ahrhunderts datierte sogenannte Vocabularius Sti. Galli erwähnt, der einen etwa 450 Wörter umfassenden Grundwortschatz überliefert, der für viele, später auch in althochdeutschen Texten bezeugte Wörter die ältesten deutschen B elege enthält. Der Vocabularius enthält Wörter für die menschlichen $\mathrm{K}$ örperteile, für $\mathrm{Him}$ mel und Erde, Witterungserscheinungen, Haustiere usw. Es liegt auf der $\mathrm{H}$ and, dass Funktion und Anlage eines Glossars auch für die Frage entscheidend sind, welche volkssprachigen Wörter überhaupt erscheinen. Ein Vogelnamenglossar in Merkversanordnung dient dem E rlernen eines lateinischen Spezialwortschatzes und benötigt dafür durchgehend die deutschen Vogelbezeichnungen als Verständnishilfe. Der Vocabularius Sti. Galli bildete für einen lateinkundigen Leser einen Zugang zu dem ihm offenbar fremden deutschen Alltagswortschatz. Das sind aber eher spezielle Fälle. 
Der viel normalere, weil sehr viel breiter überlieferte Fall sind beispielsweise die Bibelglossare, in denen wie in einem modernen Stellenkommentar fortlaufend das Textverständnis fördernde Erklärungen stehen, und zwar selbstverständlich ganz überwiegend lateinische Erklärungen. Die Volkssprachigkeit einer Erklärung bildet eher den Ausnahmefall, der zuweilen durch einen Hinweis auf die Wahl der Volkssprache wie thiudisce oder vulgariter (vgl. I. Reif fenstein 1997 mit weiterer Literatur) hervorgehoben wird.

Es ist also in Textglossaren wie übrigens auch in Textglossierungen nicht selbstverständlich, dass die Glossierung auf Deutsch erfolgt. Es ist aber auch nicht leicht erkennbar, wann und warum sie auf Deutsch erfolgt, so naheliegend eine volkssprachige Glosse bei einer Tier- oder Pflanzenbezeichnung auch erscheinen mag. Zuverlässige Einschätzungen der Motivation volkssprachiger Glossierung sind eigentlich nur zu gewinnen, wenn auch die lateinischen Teile eines Glossars bzw. einer Textglossierung untersucht sind, was erst einmal ihre E dition voraussetzen würde. Diese Voraussetzungen sind überhaupt nur in Einzelfällen gegeben.

Nur vor dem breiteren Hintergrund einer Zuweisung aller glossierten Lemmata zu onomasiologischen $\mathrm{K}$ ategorien ließe sich dann feststellen, in welchen Bereichen signifikant öfter volkssprachige Glossen gewählt werden, ohne dass damit bereits Gründe dafür erkennbar sein müssen. Es dürfte wohl einleuchten, dass hier eigentlich Voraussetzungen für die Überlieferung des volkssprachigen Wortschatzes unbekannt bleiben. Diese Beobachtung führt zu weiteren methodischen Problemen althochdeutscher Onomasiologie und kulturgeschichtlicher Lexikologie.

\section{Methodische Probleme}

Die methodisch naive ,W örter-und Sachen'-Forschung älterer Zeit ist in den jüngeren Arbeiten von einer methodisch reflektierten onomasiologischen Ansatzweise abgelöst worden, in der als dritte Größe neben Wort und Sache der Begriff steht. Autorinnen wie A. M ikel eit is-W inter (2001) oder D. H üpper -D r öge (1981 u. a.) haben natürlich, ihren Reichmann' gelesen, will sagen O. R eichmanns ,Germanistische Lexikologie' (1976) oder seinen hier schon zitierten Artikel ,H istorische Lexikologie' im HSK-Band 'Sprachgeschichte'. Daraus sei eine wichtige theoretische Einschränkung zitiert (1998), S. 612: 
Die theoretischen Grenzen der Wort- und Sachforschung liegen in ihrem auf Klassen exophorisch nachweisbarer (= konkreter) Gegenstände und Vorgänge beschränkten und selbst dort problematischen Sachbegriff, ferner in der Tatsache, daß zwischen Sache und Begriff, falls dieser überhaupt angesetzt wird, eine direkte Entsprechungsrelation unterstellt wird, in der Nichtberücksichtigung aller strukturellen Relationen des Wortschatzes, in der Alleinberücksichtigung der Darstellungsfunktion der Sprache, umgekehrt ausgedrückt in der Nichtberücksichtigung ihrer kognitiven, kommunikativen und symptomatischen Funktion [...] In der wissenschaftlichen Praxis schlägt die einfache und deshalb sehr suggestive theoretische Grundlage der Wort- und Sachforschung vor allem dann durch, wenn theoretisch eingeführte Differenzierungen, vor allem der Ansatz einer Größe ,Bedeutung/Begriff/ $N$ orstellung', in den empirischen Teilen vergessen werden und wenn diese Größe unter der $\mathrm{H}$ and mit der Sache gleichgesetzt und als sprecherunabhängige Gegebenheit in die außersprachliche Wirklichkeit projiziert wird ...

Diese generelle Gefahr kann vielleicht vermieden werden, wenn es nicht um die Bezeichnungen konkreter Gegenstände und V orgänge geht, sondern beispielsweise um Bezeichnungen sozialer, religiöser oder anderer abstrakter Gegebenheiten, bei denen man ohne die Größe ,Begriff‘ gar nicht auskommt, wie zum B eispiel bei den Bezeichnungen für das K onzept ,bekehren'. Freilich treten hier dann ganz andere methodische Probleme auf. O. R eich manns HSK -Artikel vermittelt eine Vorstellung davon, indem er über die klassische Ausprägung der Semasiologie und Onomasiologie hinaus strukturalistische und handlungstheoretische Ansätze darstellt und sein Ko-Autor D. Wolf im 5. Abschnitt, Bausteine zur Theorie einer handlungstheoretisch-konstruktivistisch begründeten historischen Lexikologie' liefert. Zwei Sätze daraus seien mit unserer Quellenlage kontrastiert (1998, S. 637):

Wortgebrauchsgeschichten, Geschichten von Wortgebrauchssystemen usw. werden auf der Grundlage von Vergleichen in allen gebrauchsrelevanten semiotischen, semantischen und pragmatischen Dimensionen der jeweiligen Wörter und Wortsysteme geschrieben. Durch kontrastive Vergleiche in allen sprachhandlungsrelevanten Dimensionen werden K ontinuitäten und Diskontinuitäten, insbesondere Innovationen, Inter- und Transferenzen unter Zuordnung zu den entsprechenden kommunikations-, interaktions-, handlungsgeschichtlichen Bedingungen festgestellt, gekennzeichnet, diskutiert, plausibilisiert, systematisiert, strukturiert bzw. typologisiert.

Für den Wortschatz der althochdeutschen Glossenüberlieferung und seine kulturgeschichtliche Auswertung sind die hier entwickelten Vorstellungen quellenbedingt unerfüllbar. Aber auch eine wesentlich bescheidenere, sozusagen naive Wörter-und Sachen-Onomasiologie stößt sehr schnell an andere methodische Grenzen. Wenn die althochdeutsche W ortschatzüberlieferung onomasiologisch ausgewertet werden soll, braucht man einen Zugang zu den historischen Sachen oder eigentlich Begriffen. Natürlich 
existieren archäologische $F$ unde von frühmittelalterlichen Sachen wie Waffen, Werkzeugen, Gerätschaften, Schmuckstücken usw. Aber ebenso natürlich haben wir keine unmittelbare Verknüpfung zur gleichzeitigen sprachlichen Realität. Das wird besonders deutlich an der klar gegliederten Arbeit von A. M ikel eit is-W int er (2001), die der sprachlichen Auswertung ein eigenes kulturhistorisches $\mathrm{K}$ apitel ,Nahrungsmittelherstellung und Speisenzubereitung im frühen Mittelalter' vorangestellt hat, in dem sie sich auf die Auswertung von archäologischem Material, von Bildquellen und von schriftlichen lateinischen Quellen aus den Bereichen Güterverwaltung und $\mathrm{K}$ losterorganisation stützt. Das althochdeutsche W ortmaterial kann diesem sachhistorischen Wissen nur über philologische Interpretation zugeordnet werden, wobei der onomasiologische Zugang zum Althochdeutschen wiederum sprachlich, eben lateinisch, vermittelt ist, nämlich über entsprechende lateinische Lemmata in Glossaren oder über lateinische Wortformen in glossierten Texten. Dabei kommen dann aber alle denkbaren textuellen Gegebenheiten wie zum Beispiel metonymischer, metaphorischer, allegorischer Wortgebrauch oder intertextuelle Beziehungen mit ins Spiel. Obendrein muss auf Seiten der Glossatoren mit ganz unterschiedlichen Funktionen der Glossierung gerechnet werden, zum Beispiel einer rein grammatischen durch bloße Angabe der Wortart, einer lexikalisch-semantischen in Form einer bloßen Gattungsangabe, zum B eispiel nux-boum, morus - boum, ornus - boum , womit die jeweilige Baumart gerade nicht identifiziert wird (E. St einmeyer /E. Siever S III, 1895, S. 490, 15; IV, 1898, S. 7, 6; III, S. 41, 58 ; vgl. H. GöTZ 1994, S. 139), oder dem Wunsch nach einem tatsächlich textstellenadäquaten Ü bersetzungsäquivalent usw. Das führt letztlich zu einer von der Überlieferung ausgehenden Vorgehensweise. Es sind die lateinischen Lemmata zu ermitteln, die für einen bestimmten Sachbereich gegeben sind; es sind die Textglossierungen im Rahmen des Verständnisses des lateinischen Textes zu analysieren, die Glossarartikel im Zusammenhang von Glossarfunktion und -anlage zu interpretieren. Dann erst stellt sich die Frage nach den historischen Sachen beziehungsweise Begriffen. H. Tief en bachs Untersuchung zu den W erkzeugbezeichnungen zeigt W ort für Wort, Beleg für Beleg dieses Vorgehen. E in W ort wie ahd. sega und seine Ablautvariante saga ist eben nicht in einem frühmittelalterlichen Bildwörterbuch überliefert, sondern zum Beispiel als V ergil-Glosse, als J esaja-Glosse usw., ohne dass aus den Stellen ohne weiteres auf die Form und die normale technische Funktion des Werkzeugs im Frühmittelalter geschlossen werden könnte $(1983$, S. 733f.). Natürlich ist auch der Weg vom althochdeut- 
schen Wort sega über seine neuhochdeutsche lautliche Entsprechung Säge zur bezeichneten mittelalterlichen Sache, Säge' ausgeschlossen.

Übrigens kommt in der ganzen über $1.300 \mathrm{H}$ andschriften umfassenden Glossenüberlieferung (vgl. R. B er gmann 1973, 1996) tatsächlich doch einmal die klassische onomasiologische Situation des Bildwörterbuchs vor, nämlich in der Pflanzenglossarüberlieferung. Hier ist die volkssprachige Bezeichnung sozusagen in das Bild der Pflanze eingetragen, nämlich in einer $\mathrm{K}$ asseler Handschrift mit dem Herbarium des Pseudo-A puleius, in der - wie E. St einmeyer sagt - die meisten Glossen „in, über oder neben dem den betr. kräuternamen illustrierenden bilde“ (III, 1895, S. 589) stehen.

\section{Schl uss}

Wenn es nach den Worten $\mathrm{O}$. R eichmanns „in der Kulturgeschichte nichts Relevantes gibt", "das nicht mit lexikalischen Mitteln bezeichnet worden wäre", dann ist der überlieferte Wortschatz jedenfalls mit der Kulturgeschichte verknüpft. Für die frühmittelalterliche Periode unserer Sprachund Kulturgeschichte liegt die Bedeutung des althochdeutschen Glossenwortschatzes dann in seiner Quantität, da zwei Drittel des althochdeutschen Wortschatzes nur hier überliefert sind, und in seiner Qualität, da durch die Spezial- und Allgemein-Glossare wie durch die Textglossierung ganz bestimmter Stellen gerade kulturgeschichtlich interessanter Wortschatz bezeugt ist. Die umfangreiche, wenn auch in der Auswahl zufällige Bearbeitung dieser Überlieferung unter kulturhistorischem Aspekt ist zunächst der Wörter-und Sachen-Tradition verpflichtet, zeigt in jüngeren Arbeiten aber durchaus ein methodisch reflektiertes onomasiologisches Vorgehen in der notwendigen Kombination mit der Semasiologie. Grundlage der historisch-lexikologischen Auswertung bleibt aber die philologische Analyse, in der auch ermittelt werden muss, in welcher Funktion überhaupt die Volkssprache bei der Glossierung verwendet wird, ehe die weitere Auswertung einsetzen kann.

\section{Liter at urverzeichnis}

A cker en, Wilhelm van (1904): Die althochdeutschen Bezeichnungen der septem peccata criminalia und ihrer filiae. Dortmund.

Al anne, Eero (1950): Die deutsche Weinbauterminologie in althochdeutscher und mittelhochdeutscher Zeit. Helsinki. 
B aur , Günter (1960): Der Wortschatz der Landwirtschaft im Bereich der ahd. Glossen. Dissertation München.

B ecker , Gertraud (1964): Geist und Seele im Altsächsischen und im Althochdeutschen. Heidelberg.

B er gmann, Rolf (1973): Verzeichnis der althochdeutschen und altsächsischen Glossenhandschriften. Mit Bibliographie der Glosseneditionen, der Handschriftenbeschreibungen und der Dialektbestimmungen. Berlin, New Y ork (Arbeiten zur F rühmittelalterforschung 6).

B er gmann, Rolf (1975): Althochdeutsche Glossen zu „Bauer“. In: Wort und Begriff „Bauer“. Zusammenfassender Bericht über die Kolloquien der Kommission für die Altertumskunde Mittel- und Nordeuropas. Herausgegeben von Reinhard W enskus, Herbert J ankuhn, Klaus Grinda. Göttingen (Abhandlungen der Akademie der Wissenschaften in Göttingen. Philologisch-Historische K lasse. Dritte Folge 89), S. 89- 127.

Ber gmann, Rolf (1996): Latin - Old High German Glosses and Glossaries. A Catalogue of Manuscripts. In: Les manuscrits des lexiques et glossaires de l'antiquité tardive à la fin du moyen âge. Actes du colloque international organisé par le Ettore Majorana Centre for Scientific Culture. Erice, 23-30 septembre 1994. Fédération Internationale des Instituts d'études Médiévales. Louvain-la-Neuve (Textes et études du Moyen Âye 4), S. 547614.

Ber gmann, Rolf (1997): Zur Textualität althochdeutscher Glossen. In: Franz Simml er (Hrsg., 1997), Textsorten und Textsortentraditionen. Bern u. a. (Berliner Studien zur Germanistik 5), S. 215-238.

Bergmann, Rolf (2000a): Ansätze zu einer Typologie der althochdeutschen Glossen- und Glossarüberlieferung. In: Theodisca. Beiträge zur althochdeutschen und altniederdeutschen Sprache und Literatur in der Kultur des früheren Mittelalters. Eine internationale Fachtagung in Schönmühl bei Penzberg vom 13. bis zum 16. März 1997. Herausgegeben von Wolfgang Haubrichs, Ernst Hel Igardt, Reiner Hildebrandt, Stephan Müller und K laus R idder . Berlin, New Y ork (Ergänzungsbände zum Reallexikon der Germanischen Altertumskunde 22), S. 77- 104.

B er gmann, R olf (2000b): Zehn St. Galler K leinigkeiten. Glossen zu allem möglichen außerhalb von Texten. In: Sprache und Dichtung in Vorderösterreich. Elsass. Schweiz. Schwaben. Vorarlberg. Tirol. Ein Symposion für Achim Masser zum 65. Geburtstag am 12. Mai 1998. Herausgegeben von Guntram A. PIangg und Eugen Thurnher. Innsbruck (Schlern-Schriften 310), S. 35-46.

Ber gmann, Rolf (2003): Volkssprachige Glossen für lateinkundige Leser? In: Sprachwissenschaft 28, S. 29-55.

Ber gmann, Rolf/G I aser, Elvira/M oul in - F ankhänel, Claudine (Hrsg., 2001): Mittelalterliche volkssprachige Glossen. Internationale Fachkonferenz des Zentrums für Mittelalterstudien der Otto-F riedrich-Universität Bamberg. 2. bis 4. August 1999. Heidelberg (Germanistische Bibliothek 13).

Björkman, Erik (1902): Die Pflanzennamen der althochdeutschen Glossen. In: ZDW 2, S. 202-233, 3, S. 263- 307.

B jör kman, Erik (1904-05): Die Pflanzennamen der althochdeutschen Glossen, II. Entlehnte Pflanzennamen. In: ZDW 6, S. 174-198.

B oor , Helmut de (1944): Zum althochdeutschen Wortschatz auf dem Gebiet der Weissagung. In: PBB 67, S. 65-110. 
$\mathrm{Br}$ andsch, J uliane (1987): Bezeichnungen für Bauern und Hofgesinde im Althochdeutschen. Mit 4 Abbildungen, Sitzungsberichte der sächsischen Akademie der Wissenschaften zu Leipzig. Philologisch-Historische K lasse, Band 127, H eft 4, Berlin.

D or nseif f, Franz (1970): Der deutsche Wortschatz nach Sachgruppen, 7. Aufl. Berlin.

Ehrismann, Gustav (1905-06): Die Wörter für ,Herr' im Althochdeutschen. In: ZDW 7, S. $173-202$.

F ehI mann, Hans-Rudolf (1982): Deutsche Heilpflanzennamen in St. Galler Handschriften (9. bis 11. J ahrhundert). In: Fachprosa-Studien. Beiträge zur mittelalterlichen Wissenschafts- und Geistesgeschichte. Herausgegeben von Gundolf K eil im Zusammenwirken mit Peter Assion, Willem Frans D aems, Heinz-UIrich R oehl . Berlin, S. 469-478.

Freudenthal , Karl Fredrik (1959): Gloria Temptatio Conversio. Studien zur ältesten deutschen Kirchensprache. Göteborg (Acta Universitatis Gothoburgensis. Göteborgs Universitets Ásskrift 65, 2, = Göteborger Germanistische F orschungen 3).

Gebhardt, Michael (1985): Die Verwaltung des königlichen Hofes im Frühmittelalter und ihre Widerspiegelung in den althochdeutschen Glossen. In: BEDSp. 5, S. 5-21.

GI aser , Elvira (2003): Typen und Funktionen volkssprachiger (althochdeutscher) Eintragungen im lateinischen Kontext. In: Sprachwissenschaft 28, S. 1-27.

G öt z, Heinrich (1994): Ü bersetzungsweisen in althochdeutschen Texten und Glossen im Spiegel eines lateinisch-althochdeutschen Glossars. In: Sprachwissenschaft 19, S. 123-164.

Gr een, Dennis H. (1965): The Carolingian Lord. Semantic Studies on F our Old High German Words. Balder, Frō, Truhtin, Hērro. Cambridge.

Groß e, Rudolf/Köppe, Ingeborg (2000): ,Kulturelles Gedächtnis' im historischen Wortschatz. Sachinformation im Althochdeutschen Wörterbuch. In: Sprachgeschichte als Textsortengeschichte. Festschrift zum 65. Geburtstag von Gotthard Lerchner. Herausgegeben von Irmhild Bar z, Ulla Fix, Marianne Schröder, Georg Schuppener. Frankfurt am Main u. a., S. 33-60.

H al I ig, Rudolf/W ar t bur g, W alther von (1963): Begriffssystem als Grundlage für die Lexikographie. 2. Aufl. Berlin.

H enkel, Nikolaus (1997): Glosse. In: Reallexikon der deutschen Literaturwissenschaft. Neubearbeitung des Reallexikons der deutschen Literaturgeschichte. Herausgegeben von Harald F r icke, K laus G r ubmül I er , J an-Dirk Müller. 1. Bd. Berlin, New Y ork, S. $727 f$.

H eyne, Moriz (1899ff.): Fünf Bücher deutscher Hausaltertümer von den ältesten geschichtlichen Zeiten bis zum 16. J ahrhundert. Leipzig, Nachdruck Düsseldorf 1985ff.

H il ker-Suckrau, Martina (1981): Sachbezeichnungen aus dem Bereich des Hausbaus im Frühmittelalter. In: Wörter und Sachen im Lichte der Bezeichnungsforschung. Herausgegeben von Ruth Schmidt-W iegand. Berlin, New York (Arbeiten zur Frühmittelalterforschung 1), S. 58-73.

H och hol zer, Rupert (1996): Himmel und Hölle. Onomasiologische und semasiologische Studien zu den J enseitsbezeichnungen im Althochdeutschen, (Regensburger Beiträge zur deutschen Sprach- und Literaturwissenschaft B 60), Frankfurt/Main u.a.

H üpper , Dagmar (1986): Buoh und scrift. Gattungen und Textsorten in frühmittelalterlichen volkssprachigen Schriftzeugnissen: Zur Ausbildung einer Begrifflichkeit. In: FMSt. 20, S. 93-122.

H üpper, Dagmar (1986): Sikihelm chaisurlih und chuninchelm. Althochdeutsche Glossen zu dem Helm als Herrschaftszeichen. In: Sprache und Recht. Beiträge zur Kulturgeschichte des Mittelalters. Festschrift für Ruth Schmidt-Wiegand zum 60. Geburtstag. Herausge- 
geben von Karl Hauck, Karl K r oeschell, Stefan Sonder egger u. a. 1. Bd. Berlin, New York, S. 284-304.

H üpper -D r öge, Dagmar (1981): Schutz- und Angriffswaffen nach den Leges und verwandten fränkischen Rechtsquellen. In: Wörter und Sachen im Lichte der Bezeichnungsforschung. Herausgegeben von Ruth Schmidt-W iegand. Berlin, New York (Arbeiten zur Frühmittelalterforschung 1), S. 107-127.

H üpper -D r öge, Dagmar (1983): Schild und Speer. Waffen und ihre Bezeichnungen im frühen Mittelalter. Frankfurt (Main) u. a. (Germanistische Arbeiten zur Kulturgeschichte 3).

H üpper -D r öge, Dagmar (1984): Der gerichtliche Zweikampf im Spiegel der Bezeichnungen für ,K ampf', ,Kämpfer', ,W affen'. In: FMSt. 18, S. 607-661.

J ones, William J ervis (1990): German Kinship Terms (750-1500). Documentation and Analysis. Berlin, New Y ork (Studia Linguistica Germanica 27).

K I opsch, Paul (1955): Notkers W ortschatz auf dem Gebiet des F ühlens. Dissertation Köln.

K ochskämper, Birgit (1999): ,Frau' und ,Mann' im Althochdeutschen. Frankfurt (Main) (Germanistische Arbeiten zur Sprache und Kulturgeschichte 37).

L e cout eux, Claude (1983): Hagazussa - Striga - Hexe. In: ÉG 38, S. 161-178.

L ecout eux, Claude (1985): Vom Schrat zum Schrättel. Dämonisierungs-, Mythologisierungsund Euphemisierungsprozeß einer volkstümlichen Vorstellung. In: Euphorion 79, S. 95108.

Lötscher, Andreas (1973): Semantische Strukturen im Bereich der althochdeutschen und mittelhochdeutschen Schallwörter. Berlin, New York (Quellen und Forschungen zur Sprach- und Kulturgeschichte der germanischen Völker. Neue Folge 53).

M aschke, Erich (1926): Studien zu W affennamen der althochdeutschen Glossen. Dissertation Greifswald.

M asser , Achim (1966): Die Bezeichnungen für das christliche Gotteshaus in der deutschen Sprache des Mittelalters. Berlin (Philologische Studien und Quellen 33).

Meineke, Birgit (1987): CHIND und BARN im Hildebrandslied vor dem Hintergrund ihrer althochdeutschen Überlieferung. Göttingen (Studien zum Althochdeutschen 9).

M eineke, Eckhard (1984): Bernstein im Althochdeutschen. Mit Untersuchungen zum Glossar Rb. Göttingen. (Studien zum Althochdeutschen 6).

Mezger , Fritz (1930): Die Gruppe ,Herr sein, Knecht sein' im Germanischen. In: Archiv für das Studium der neueren Sprachen 158, S. 96- 98.

Mikel eit is-W inter, Almut (2001): Der Bereich Nahrungszubereitung im althochdeutschen Wortschatz. Onomasiologisch-semasiologische Untersuchungen. Berlin (Althochdeutsches Wörterbuch. Beiband).

M unske, Horst Haider (1973): Der germanische Rechtswortschatz im Bereich der Missetaten. Philologische und sprachgeographische Untersuchungen, I: Die Terminologie der älteren westgermanischen Rechtsquellen. Berlin, New Y ork (Studia Linguistica Germanica 8/1).

N euss, Elmar (1973): Studien zu den althochdeutschen Tierbezeichnungen der Handschriften Paris lat. 9344, Berlin lat. $8^{\circ} 73$, Trier R. III. 13 und Wolfenbüttel 10. 3. Aug. $4^{\circ}$. München (Münstersche Mittelalter-Schriften 16).

$N$ ieder hel I mann, Annette (1981): H eilkundliches in den Leges. Die Schädelverletzungen und ihre Bezeichnungen. In: Wörter und Sachen im Lichte der Bezeichnungsforschung, herausgegeben von Ruth Schmidt-W iegand. Berlin, New York (Arbeiten zur Frühmittelalterforschung 1), S. 74-90. 
Nieder hel I mann, Annette (1983): Arzt und Heilkunde in den frühmittelalterlichen Leges. Eine wort- und sachkundliche Untersuchung. Berlin, New York (Arbeiten zur F rühmittelalterforschung 12).

OI ber g, Gabriele von (1983): Freie, Nachbarn und Gefolgsleute. Volkssprachige Bezeichnungen aus dem sozialen Bereich in den frühmittelalterlichen Leges. Frankfurt (Main) u. a. (Germanistische Arbeiten zu Sprache und Kulturgeschichte 2).

Ol ber g, Gabriele von (1991): Die Bezeichnungen für soziale Stände, Schichten und Gruppen in den Leges Barbarorum. Berlin, New York. (Die volkssprachigen Wörter der Leges Barbarorum 2. Arbeiten zur F rühmittelalterforschung 11).

Pal ander, Hugo (1899): Die althochdeutschen Tiernamen, I. Die Namen der Säugetiere, Darmstadt.

Paul y, L. (1944): Das Begriffsfeld des Zweifels in der althochdeutschen und altsächsischen Literatur sowie in der Geistlichendichtung der Cluniazenserzeit, Dissertation F reiburg i. $\mathrm{Br}$. (Maschinenschriftlich).

Reichmann, Oskar (1976): Germanistische Lexikologie. Zweite, vollständig umgearbeitete Auflage von "Deutsche W ortforschung". Stuttgart (Sammlung Metzler 82).

R eichmann, Oskar/W olf, Dieter (1998): Historische Lexikologie. In: Sprachgeschichte. Ein Handbuch zur Geschichte der deutschen Sprache und ihrer Erforschung. 2. Auflage herausgegeben von Werner B esch, Anne B et t en, Oskar R eichmann, Stefan Sonder egger . Berlin, New Y ork (HSK 2.1.), S. 610-643.

R eif f enst ein, Ingo (1997): theodiscus in den althochdeutschen Glossen. In: Grammatica ianua artium. Festschrift für Rolf Bergmann zum 60. Geburtstag. Herausgegeben von Elvira GI aser und Michael Schl aef er. Heidelberg, S. 71-84.

R ein, Matthias (2001): Zur Bedeutungsbreite des Wortes bekehren und zur Bezeichnungskonkurrenz im Rahmen des K onzeptes >bekehren<im früheren Althochdeutschen. In: ZLL 31, S. 7-79.

R el I eke, Walburga (1980): E in Instrument spielen. Instrumentenbezeichnungen und Tonerzeugungsverben im Althochdeutschen, Mittelhochdeutschen und Neuhochdeutschen, Heidelberg (Monographien zur Sprachwissenschaft 10).

R iecke, J örg (2000): Anatomisches und Heilkundliches in altsächsischen Glossaren. In: Speculum Saxonum. Studien zu den kleineren altsächsischen Sprachdenkmälern. Herausgegeben von Arend Quak, ABÄG 52, S. 207-225.

$R$ iecke, J örg (2003): Über die volkssprachigen Anfänge des Schreibens über K örper, Krankheit, Heilung. Aspekte der Frühgeschichte der medizinischen Fachsprache im Deutschen. In: Sprachwissenschaft 27, S. 245-271.

$\mathrm{R}$ iecke, J örg (2004): Die F rühgeschichte der mittelalterlichen medizinischen $\mathrm{F}$ achsprache im Deutschen I-II. Berlin, New York.

R ohr , J ohannes (1909): Die Gefäße in den althochdeutschen Glossen. Dissertation Greifswald.

Rooth, Erik (1961): Zu den Bezeichnungen für ,Eiszapfen' in den germanischen Sprachen. Historisch-wortgeographische und etymologische Studien, Kungl. Vitterheds Historie och Antikvitets A kademiens Handlingar. Stockholm (Filologisk-Filosofiska Serien 8).

Rupr echt, Dietrich (1959): Tristitia. Wortschatz und Vorstellung in den althochdeutschen Sprachdenkmälern. Göttingen (Palaestra 227).

Schmidt-W iegand, Ruth (1975): Historische Onomasiologie und Mittelalterforschung. In: F rühmittelalterliche Studien 9, S. 49-78. 
Schmidt-W iegand, Ruth (1981): Wörter und Sachen. Zur Bedeutung einer Methode für die Frühmittelalterforschung: Der Pflug und seine Bezeichnungen. In: Wörter und Sachen im Lichte der Bezeichnungsforschung, herausgegeben von Ruth Sch midt -W iegand. Berlin, New Y ork (Arbeiten zur Frühmittelalterforschung 1), S. 1-41.

Schröder , Edward (1918a): Studien zu den deutschen Münznamen, I. Scherf. In: ZVSp. 48, S. 141- 150 .

Schr öder , Edward (1918b): Studien zu den deutschen Münznamen, II. Pfennig, III. Schilling, IV. Schatz. In: ZVSp. 48, S. 241-275.

Schützeichel, Rudolf (1982): Addenda und Corrigenda zu Steinmeyers Glossensammlung. Göttingen (Nachrichten der Akademie der Wissenschaften in Göttingen. I. PhilologischHistorische K lasse. J ahrgang 1982, Nr. 6).

Schützeichel, Rudolf (1985): Addenda und Corrigenda (II) zur althochdeutschen Glossensammlung. Göttingen (Studien zum Althochdeutschen 5).

Schützeichel, Rudolf (1991): Addenda und Corrigenda (III) zum althochdeutschen Wortschatz. Göttingen (Studien zum Althochdeutschen 12).

Smet, Gilbert A.R. de (1953): Die altdeutschen Bezeichnungen des Leidens Christi. In: PBB 75 (Halle) S. 273-296. Wiederabgedruckt in: Gilbert A.R. de Smet, Kleine deutsche Schriften, S. 13-36.

Smet, Gilbert A.R. de (1961): Auferstehen und Auferstehung im Althochdeutschen. In: PBB 82 (Halle) S. 175-198. Wiederabgedruckt in: Gilbert A.R. de Smet, Kleine deutsche Schriften, S. 48-71.

Smet, Gilbert A.R. de (1962): Die Ausdrücke für „leiden“ im Althochdeutschen. In: Wirkendes Wort. Sammelband II. Ältere deutsche Sprache und Literatur. Düsseldorf. Wiederabgedruckt in: Gilbert A.R. de Smet, K leine deutsche Schriften, S. 37- 47.

Smet, Gilbert A.R. de (1973). Remissio peccatorum. Vergeben und Sündenvergebung im Althochdeutschen. In: Festschrift für Ingeborg Schröbler zum 65. Geburtstag. Herausgegeben von Dietrich Schmidt ke, Helga Schüppert. PBB 95 Sonderheft. Tübingen, S. 1-22. Wiederabgedruckt in: Gilbert A.R. de Smet, K leine deutsche Schriften, S. 108-129.

Spl et t, J ochen (2000): Lexikologie und Lexikographie des Althochdeutschen. In: Sprachgeschichte. Ein Handbuch zur Geschichte der deutschen Sprache und ihrer Erforschung, 2. Auflage herausgegeben von Werner Besch, Anne B etten, Oskar Reichmann, Stefan Sonder egger. Berlin, New Y ork. (HSK 2.2.), S. 1196-1206.

Staiti, Chiara (2000): Das Wortfeld der superbia in der frühdeutschen Überlieferung. In: Theodisca. Beiträge zur althochdeutschen und altniederdeutschen Sprache und Literatur in der Kultur des frühen Mittelalters. Eine internationale Fachtagung in Schönmühl bei Penzberg vom 13. bis zum 16. März 1997. Herausgegeben von Wolfgang $\mathrm{H}$ aubrichs, Ernst H el I gardt, Reiner H il debr andt, Stephan M ül I er, Klaus Ridder. Berlin, New York (Ergänzungsbände zum Reallexikon der Germanischen Altertumskunde 22), S. $143-188$.

St einmeyer , Elias/Siever s, Eduard (1879-1922): Die althochdeutschen Glossen, I-V, Nachdruck Dublin, Zürich 1968- 1969.

St ricker, Stefanie (2001): Zu den Erträgen einer Werktypologisierung für eine Funktionsbestimmung am Beispiel der Versus de volucribus. In: Mittelalterliche volkssprachige Glossen. Internationale Fachkonferenz des Zentrums für Mittelalterstudien der Otto-F riedrich-Universität Bamberg 2. bis 4. August 1999. Herausgegeben von Rolf B er g mann, EIvira Gl aser, Claudine M oul in-F ankhänel. Heidelberg (Germanistische Bibliothek 13), S. 51-573. 
Suol ahti, Hugo (1909): Die deutschen Vogelnamen. Eine wortgeschichtliche Untersuchung, Straßburg.

Thoma, Herbert (1958): Glossen, althochdeutsche. In: Reallexikon der deutschen Literaturgeschichte. 2. Aufl., herausgegeben von Werner $\mathrm{K}$ ohl schmidt und Wolfgang M ohr. 1. Bd. Berlin, New York, S. 579-589.

Tief enbach, Heinrich (1980a): Bezeichnungen für Mist und Dünger im Althochdeutschen. In: Untersuchungen zur eisenzeitlichen und frühmittelalterlichen Flur in Mitteleuropa und ihrer Nutzung. Bericht über die Kolloquien der Kommission für die Altertumskunde Mittel- und Nordeuropas in den J ahren 1975 und 1976. Herausgegeben von Heinrich Beck, Dietrich Denecke, Herbert Jankuhn. 2. Bd. Mit 49 Abbildungen und 15 Tabellen. Göttingen (Abhandlungen der Akademie der Wissenschaften in Göttingen. PhilologischHistorische K lasse. Dritte Folge 116), S. 45-54 .

Tief enbach, Heinrich (1980b): Bezeichnungen für Fluren im Althochdeutschen, Altsächsischen und Altniederfränkischen. In: Untersuchungen zur eisenzeitlichen und frühmittelalterlichen Flur in Mitteleuropa und ihrer Nutzung. Bericht über die Kolloquien der Kommission für die Altertumskunde Mittel- und Nordeuropas in den J ahren 1975 und 1976. Herausgegeben von Heinrich Beck, Dietrich D enecke, Herbert J ankuhn. 2. Bd.. Mit 49 Abbildungen und 15 Tabellen. Göttingen (Abhandlungen der Akademie der Wissenschaften in Göttingen. Philologisch-Historische Klasse. Dritte Folge 116), , S. 287322.

Tief enbach, Heinrich (1983): Bezeichnungen für Werkzeuge aus dem Bauhandwerk im Althochdeutschen. In: Das Handwerk in vor- und frühgeschichtlicher Zeit. Bericht über die Kolloquien der Kommission für die Altertumskunde Mittel- und Nordeuropas in den J ahren 1977 bis 1980. Herausgegeben von Herbert J ankuhn, Walter J anssen, Ruth Schmidt-W iegand, Heinrich Tief enbach. 2. Bd. Archäologische und philologische Beiträge. Mit zahlreichen Abbildungen. Göttingen (Abhandlungen der Akademie der Wissenschaften in Göttingen. Philologisch-Historische K lasse. D ritte F olge 123), S. 717- 750.

Tief enbach, Heinrich (1989): Furtnamen und Verwandtes. In: Untersuchungen zu Handel und Verkehr der vor- und frühgeschichtlichen Zeit in Mittel- und Nordeuropa, V. Der Verkehr. Verkehrswege, Verkehrsmittel, Organisation. Bericht über die K olloquien der Kommission für die Altertumskunde Mittel- und Nordeuropas in den J ahren 1980 bis 1983. Herausgegeben von Herbert J ankuhn,Wolfgang K immig, Else Ebel. Mit $118 \mathrm{Ab}-$ bildungen, Göttingen (Abhandlungen der Akademie der Wissenschaften in Göttingen. Philologisch-Historische K lasse. Dritte Folge 180), S. 262-290.

Trier , J ost (1931): Der deutsche Wortschatz im Sinnbezirk des Verstandes. Die Geschichte eines sprachlichen F eldes, I: Von den Anfängen bis zum Beginn des 13. J ahrhunderts. Heidelberg.

Voetz, Lothar (1983): Handwerkerbezeichnungen auf -man im Althochdeutschen. In: Das Handwerk in vor- und frühgeschichtlicher Zeit. Bericht über die Kolloquien der Kommission für die Altertumskunde Mittel- und Nordeuropas in den J ahren 1977 bis 1980. Herausgegeben von Herbert J ankuhn, Walter J anssen, Ruth Schmidt -W iegand, Heinrich Tief enbach. 2. Bd. Archäologische und philologische Beiträge. Mit zahlreichen Abbildungen. Göttingen (Abhandlungen der Akademie der Wissenschaften in Göttingen. Philologisch-Historische K lasse. Dritte Folge 123), S. 642-669.

V oet z, Lothar (1989): Zu den zentralen W egbezeichnungen im Althochdeutschen. In: Untersuchungen zu Handel und Verkehr der vor- und frühgeschichtlichen Zeit in Mittel- und Nordeuropa, V. Der Verkehr. Verkehrswege, Verkehrsmittel, Organisation. Bericht über 
die Kolloquien der Kommission für die Altertumskunde Mittel- und Nordeuropas in den J ahren 1980 bis 1983. Herausgegeben von Herbert J ankuhn, Wolfgang K immig, Else E bel . Mit 118 Abbildungen, (Abhandlungen der Akademie der Wissenschaften in Göttingen. Philologisch-Historische K lasse. Dritte F olge 180). Göttingen, S. 229-261.

W aag, Anatol (1931-32): Die Bezeichnungen des Geistlichen im Althoch- und Altniederdeutschen. Eine wortgeschichtliche und wortgeographische Untersuchung. In: Teuthonista 8, S. 1-54.

W esche, Heinrich (1932): Das Heidentum in der althochdeutschen Sprache, I. Die Kultstätte. Dissertation Göttingen.

W esche, Heinrich (1937): Beiträge zu einer Geschichte des deutschen Heidentums. In: PBB 61, S. 1-116.

W esche, Heinrich (1940): Der althochdeutsche Wortschatz im Gebiete des Zaubers und der Weissagung, (Untersuchungen zur Geschichte der deutschen Sprache 1). Halle.

Zanier , Tiziana (1989/90): II lessico alimentare nelle glosse antico alto tedesche, Dissertation Udine. 